\title{
Article \\ Joule-Level Twelve-Pass LD End-Pumped Bonded Neodymium Glass Laser Amplifier
}

\author{
Long Pan ${ }^{1,2}\left(\right.$, Shengzhe $\mathrm{Ji}^{1,2}{ }^{10}$, Wenfa Huang ${ }^{1, *}$, Jiangtao Guo $^{1}{ }^{1}$, Xinghua Lu ${ }^{1}$, Jiangfeng Wang ${ }^{1}$, Wei Fan ${ }^{1}$, \\ Xuechun $\mathrm{Li}^{1}$ and Jianqiang Zhu ${ }^{1}$ \\ 1 Joint Laboratory on High Power Laser and Physics, Shanghai Institute of Optics and Fine Mechanics, \\ Chinese Academy of Sciences, Shanghai 201800, China; panlong1@siom.ac.cn (L.P.); szji@siom.ac.cn (S.J.); \\ guojiangtao@siom.ac.cn (J.G.); luxingh@foxmail.com (X.L.); wajfeng@163.com (J.W.); \\ fanweil@siom.ac.cn (W.F.); lixuechun@siom.ac.cn (X.L.); jqzhu@mail.shcnc.ac.cn (J.Z.) \\ 2 Center of Materials Science and Optoelectronic Engineering, University of Chinese Academy of Sciences, \\ Beijing 100049, China \\ * Correspondence: huangwf@siom.ac.cn; Tel.: +86-216-991-8164
}

Citation: Pan, L.; Ji, S.; Huang, W.; Guo, J.; Lu, X.; Wang, J.; Fan, W.; Li, X.; Zhu, J. Joule-Level Twelve-Pass LD End-Pumped Bonded Neodymium Glass Laser Amplifier. Photonics 2021, 8, 96 .

https://doi.org/10.3390/ photonics 8040096

Received: 25 February 2021

Accepted: 26 March 2021

Published: 30 March 2021

Publisher's Note: MDPI stays neutral with regard to jurisdictional claims in published maps and institutional affiliations.

Copyright: (C) 2021 by the authors. Licensee MDPI, Basel, Switzerland. This article is an open access article distributed under the terms and conditions of the Creative Commons Attribution (CC BY) license (https:// creativecommons.org/licenses/by/ $4.0 /)$

\begin{abstract}
This paper reports on a Joule-level multi-pass laser amplification device with diode endpumped square-rod neodymium glass (Nd:glass) bonded to K9 glass. The device generated 1.17 J pulse energy at $1 \mathrm{~Hz}$ and $1053 \mathrm{~nm}$. The optical-to-optical efficiency was 13.01\%, and the effective energy extraction efficiency was $44.23 \%$. Comparing Nd:glass of the same specification without K9 glass under the same conditions, the thermal wave aberration of the former was $85.71 \%$ of that of the latter, which is $0.78 \mathrm{um}$. The near-field modulation degree at the highest energy output was 1.42 within $90 \%$ of the spot, and the far-field energy concentration was $81.88 \%$ within the 2.5-fold diffraction limit. The Nd:glass bonding method of the square rod is relatively novel in laser amplification systems pumped by the diode end face and can be further studied in future works.
\end{abstract}

Keywords: joule-level; neodymium glass; diode end-pumped; bonded

\section{Introduction}

Solid-state laser systems with high energy and high repetition rates have attracted wide attention. In China's SG (Shen Guang) Up facility [1] and the US National Ignition Facility (NIF) [2], the preamplification module is a Joule-level solid-state laser amplification device. High-energy, intense, and high-repetition rate laser has a wide range of applications in astrophysics, plasma jets, and other high-energy density physics [3-5]. In terms of engineering applications, these laser systems have a lot of value. It is used as a pump source in chirped pulse amplification (CPA) or optical parametric chirped pulse amplification (OPCPA) systems [6-8]. In laser shock peening [9], laser-induced damage threshold measurement [10] and other materials processing also have great potential. However, in current application scenarios, many laser systems use flashlamp pumping methods, which have high thermal effects and low electrooptical conversion efficiency. The emission spectrum of a laser diode has a central wavelength of $802 \mathrm{~nm}$ and a narrow full width at half maximum (FWHM) of $3 \mathrm{~nm}$, which can be well-matched with the Nd:glass with absorption wavelength of $802 \mathrm{~nm}$ and FWHM of $14 \mathrm{~nm}$. In this case, the small heat sink and high electrooptical conversion efficiency are suitable for laser systems with high repetition rates. Therefore, laser diode pumped solid-state lasers have had some good reports in recent years. A DiPOLE (Diode Pumped Optical Laser Experiment) laser has achieved $105 \mathrm{~J}$ output energy based on diode pumping at a repetition frequency of $10 \mathrm{~Hz}$, and the average power has reached the $\mathrm{kW}$ level [11,12]. A laser system with an output energy of $9.3 \mathrm{~J}$ and a repetition frequency of $33.3 \mathrm{~Hz}$ has been reported [13]. Other projects, such as POLARIS (Petawatt Optical Laser Amplifier for Radiation Intensive Experiments) in Germany and HAPLS (High-repetition-rate Advanced Petawatt Laser System) in the United States, have achieved output energy of tens of Joules at multiple repetition frequencies [14,15]. 
Neodymium phosphate glass (Nd:glass) lasers currently have distinctive features among Joule-level lasers. They have a higher level of energy storage density, lower quantum defects, and can be produced in large sizes. However, due to the lower thermal conductivity, Nd:glass is limited in the repetitive frequency laser systems, and a better heat dissipation strategy is required. In order to have a smaller thermal effect, the structure of the plate gain medium is generally used. Huang et al. demonstrated the composite plate technology of a sapphire cooling plate for $\mathrm{Nd}$ :glass lasers, and the relay imaging multi-pass technology obtained $560 \mathrm{~mJ}$ output energy at $1 \mathrm{~Hz}$ [16]. Recently, Yao et al. used square-rod $\mathrm{Nd}$ :glass lasers to achieve a $1 \mathrm{~Hz}$ output energy in a $1 \mathrm{~J}$ system under LD (laser diode) pumping [17], but the effective energy extraction efficiency is not high, only $12 \%$. The cooling of rod-shaped Nd:glass lasers generally adopts side-side cooling, but if it is based on end-face pumping, the heat generated due to quantum defects and other reasons is mainly concentrated at the end surface of the gain medium, and the thermal effect is more obvious. Therefore, a solution for end-face bonding glass was proposed.

The current bonding technology has several methods, including surface-activated bonding [18], chemically activated direct bonding [19], and thermal diffusion bonding [20]. The method of atomic diffusion bonding has also been reported [21]. Surface-activated bonding is to treat the bonding surface with acetone or other solutions and then irradiate it with a fast atom beam to form suspending bonds, but this method is more expensive to process. Chemical surface activation bonding uses a strong acid and a strong base to act on the bonding surface and then optically connect them to form a stable bonding structure. Someone reported a kind of thermal diffusion bonding in which the oxide layer of the crystal is removed after surface treatment and a phosphate glass layer of several nanometers in thickness is formed [22]. After multi-stage heating (the highest temperature reaches above $1000{ }^{\circ} \mathrm{C}$ ), the part diffuses to the bonded crystal in the formation of a strong bonding layer. As for Nd:glass, its bonding ability cannot reach the diffusion level within its acceptable temperature range. For Nd:glass, the degree of diffusion of end-face molecules in its endurable temperature range is weaker than that of other crystals. The Nd:glass bonding method mentioned in this article is a thermal bonding method based on thermal diffusion bonding, and the heating temperature is about $100^{\circ} \mathrm{C}$.

In this paper, a technical scheme of diode end-pumping a square-rod Nd:glass endface bonding K9 glass laser is proposed, which realizes the Joule-level amplification with high light efficiency and high effective energy extraction efficiency. Due to the same substrate between Nd:glass and K9 glass, the thermal expansion coefficient, refractive index, and other parameters are basically the same, which ensures transmittance of the gain medium and avoids the separation of the bonding surface. The experiment shows that the transmittance of bonded Nd:glass is $99.53 \%$, and the Fresnel diffraction at the bonding surface is very small. The thermal conductivity of K9 glass is three times that of Nd:glass. In the case of end-face pumping, part of the heat is conducted through $\mathrm{K} 9$ glass, which can reduce the heat density of the entire gain medium. In the experiment, compared with unbonded glass in the same state, the thermal wavefront aberration of the former is $85.71 \%$ of that of the latter. Under the diode end-pumping of $9.02 \mathrm{~J}$ at $802 \mathrm{~nm}$, the relay imaging multi-pass amplification technology is adopted to achieve twelve-pass amplification, and the output energy is $1.17 \mathrm{~J}$ at $1 \mathrm{~Hz}$ and $1053 \mathrm{~nm}$. The optical-to-optical efficiency is $13.01 \%$, and the effective energy extraction efficiency is $44.23 \%$. The twelvepass near-field modulation is 1.42 within $90 \%$ of the spot range. The far-field energy concentration is $81.88 \%$ within 2.5 times the diffraction limit.

\section{Experimental Setup}

\subsection{Amplification System Setup}

The schematic diagram of the laser diode end-pump-bonded Nd:glass multi-pass laser amplifier system is shown in Figure 1. The laser amplifier system mainly includes a pump system, a beam expander (BE), a serrated aperture (SA), a polarization beam splitter (PBS), a half-wave plate $(\lambda / 2)$, two $45^{\circ}$ Faraday rotators (FR1 and FR2), a Pockels cell (PC), two 
thin-film polarizers (P1 and P2), two sets of 4F relay imaging vacuum telescope systems (VT1 and VT2), and a bonded Nd:glass laser amplifier head (AMP), as well as some mirrors (M1, M2, M3, TRM1, and TRM2). The pump system includes two sets of pump-coupling optical paths, which pump the two end faces of the laser amplifier head vertically. The LD array in each group of coupled optical paths is composed of 60 closely-arranged bars, with an emission area of $11 \mathrm{~cm} \times 1.5 \mathrm{~cm}$. Each LD array has a maximum pump power of $20 \mathrm{~kW}$ at a wavelength of $802 \mathrm{~nm}$. The beam is smoothed through the lens group, and finally the two sets of coupled optical paths are aligned in the middle of the laser amplifier head to form an $8 \mathrm{~mm} \times 8 \mathrm{~mm}$ square flat-top pump spot, which is also the position of the image plane of the two pump spots. The pump distribution is shown in Figure 2. The pump distribution measured on the image plane maintains high uniformity in both the horizontal and vertical directions, and the spatial intensity modulation within the entire platform is less than 15\%. Both VT1 and VT2 are composed of planoconcave lenses with a focal length of $750 \mathrm{~mm}$. The central section of AMP is on their image plane (object plane) and the three end mirrors M1, M2, and M3 also have their image plane positions respectively to maintain the beam quality during the beam transmission. A $5 \mathrm{~mm}$ diameter pinhole plate is installed at the focal point of VT1 to filter and block high-frequency spatial light and some stray light.

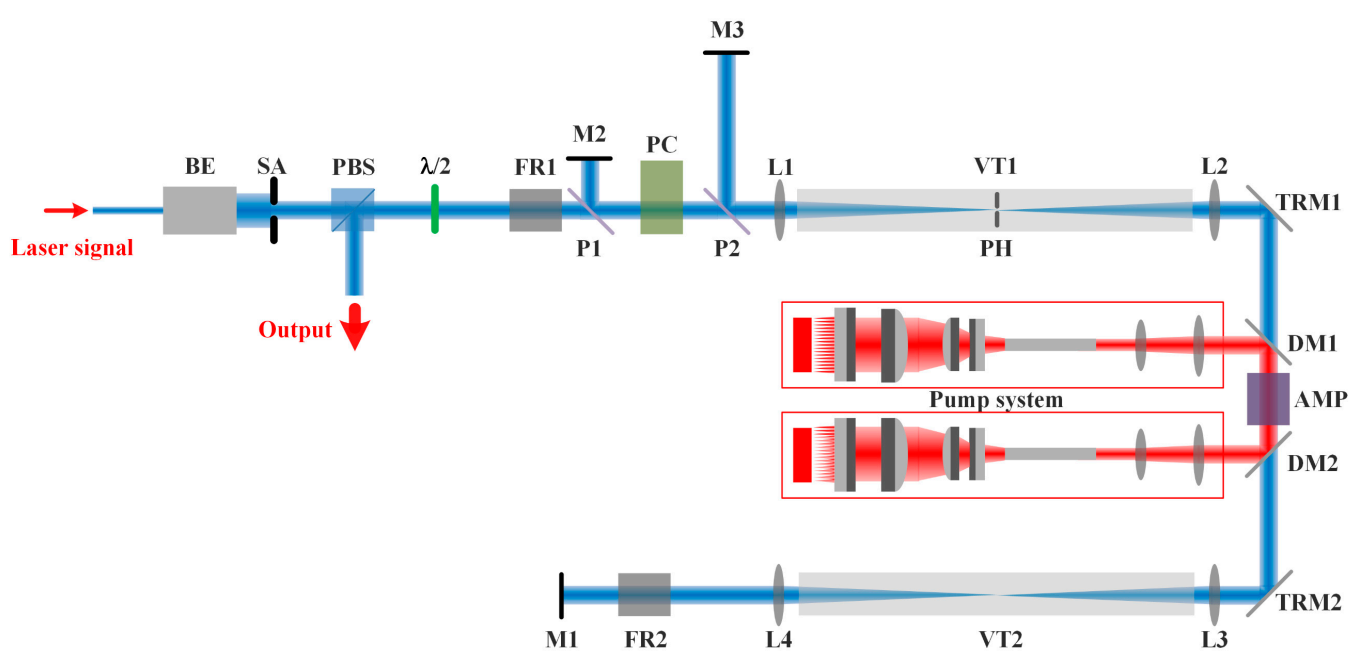

Figure 1. LD end-pumped Nd:glass multi-pass laser amplifier system. BE: beam expander; SA: serrated aperture; PBS: polarization beam splitter; $\lambda$ /2: half-wave plate; FR1 and FR2: Faraday rotators; PC: Pockels cell; P1 and P2: thin-film polarizers; M1, M2, and M3: end mirrors; L1, L2, L3, and L4: convex lenses with $\mathrm{f}=750 \mathrm{~mm}$; TRM1 and TRM2: totally reflective mirrors; DM1 and DM2: dichroic mirrors; VT1 and VT2: vacuum tubes; PH: pinhole plate; AMP: bonded Nd:glass amplifier head.

The input signal laser is a 1053-nm pulse laser with a pulse width of 5 ns generated by a regenerative amplifier with a repetition frequency of $1 \mathrm{~Hz}$. After passing through a $5 \times$ beam expander and an $8-\mathrm{mm}$ square sawtooth aperture, the signal light is spatially shaped from a circular Gaussian beam into an 8-mm square flat-top beam. Subsequently, the laser passes through the PBS, an isolator composed of $\lambda / 2$ and FR1, and P1, its polarization state is $\mathrm{P}$ polarization, and it enters the traditional four-pass amplification optical path. When the laser passes through the PC for the first time, the power of the PC is turned off. Due to the polarization control effect of FR2, the laser goes to the fourth power in the optical path and is coupled out from P2. Before the laser passes through the PC for the second time, the power of the PC is turned on and the voltage is adjusted to a half-wave voltage. At this time, the PC is used as a half-wave plate. After passing through the PC, the laser polarization state becomes $S$ polarization, passing through the M2 mirror, and passing through the energized PC for the third time, the laser polarization state becomes $\mathrm{P}$ polarization and continues to be amplified four times in the optical path, and then the laser 
is coupled out from P2. The power is turned off, and the laser passes through the PC for the fourth time, and the eight-pass amplified laser is coupled out from the PBS. In order to achieve twelve-pass magnification, when the laser passes through the PC for the fourth and fifth times, the PC is kept energized, so that the laser performs an additional four-pass magnification in the optical path. Before passing the PC for the sixth time, the power of the PC is turned off, and the twelve-pass amplified laser is coupled out from the PBS.

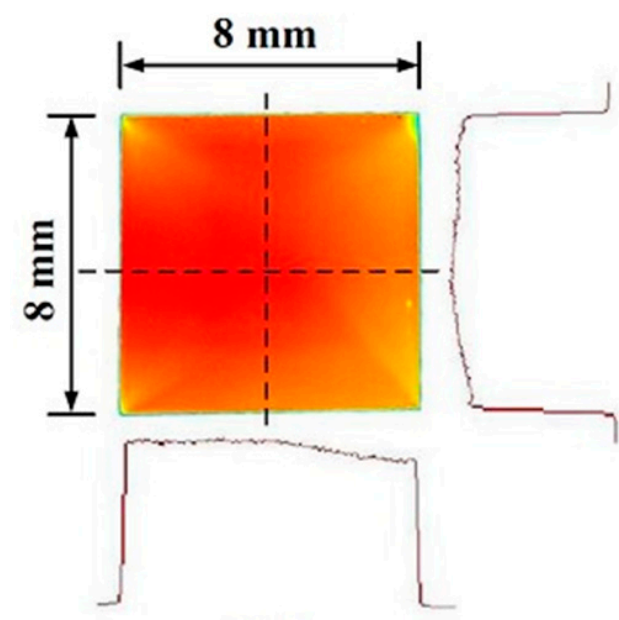

Figure 2. Pump light distribution.

\subsection{Amplifier Head}

The amplifier head is side-cooled by circulating water at $23{ }^{\circ} \mathrm{C}$ as shown in Figure 3 . Figure $3 \mathrm{a}$ is an assembly drawing of the amplifier head; Figure $3 \mathrm{~b}$ is a cross-sectional view of it and it is sealed with O-rings on both sides of the square bar.
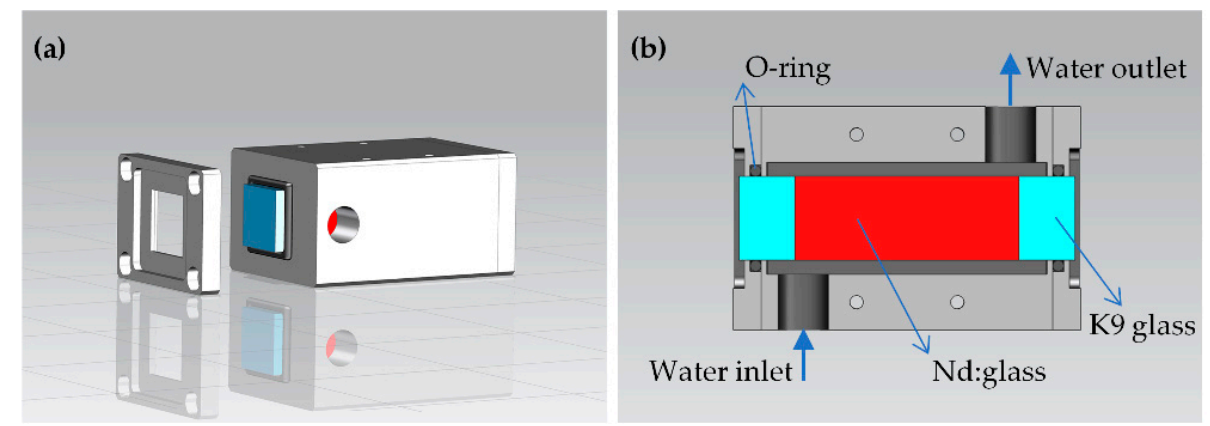

Figure 3. Amplifier head. (a) Structure diagram; (b) sectional view.

The gain medium of the laser amplifier head is a bonded Nd:glass square rod with a size of $15 \mathrm{~mm} \times 15 \mathrm{~mm} \times 60 \mathrm{~mm}$, and the gain zone is $0.5 \mathrm{wt} . \%$ doped Nd:glass with a length of $40 \mathrm{~mm}$, and the two end faces are bonded separately with 10-mm thick K9 glass. The Nd:glass type N31 was independently made and processed by the Shanghai Institute of Optics and Fine Mechanics (SIOM). Its density is $2.53 \mathrm{~g} / \mathrm{cm}^{3}$, the stimulation emission cross-section is $3.8 \times 10^{-20} \mathrm{~cm}^{2}$, and the fluorescence lifetime is $351 \mu \mathrm{s}$. The substrate of Nd:glass is $\mathrm{K} 9$ glass, and some physical parameters of the two materials such as refractive index (1.53) and thermal expansion coefficient $\left(1.07 \times 10^{-5} / \mathrm{K}\right)$ are consistent, which reduces the laser transmission loss. It also avoids the separation of the bonding surface caused by the thermal deformation of the medium during the pumping process. The two end faces of the bonded square rod correspond to the antireflection coatings of the pump wavelength and the laser wavelength, and the transmittance of the bonded square rod is $99.53 \%$ at a wavelength of $1053 \mathrm{~nm}$. 
As shown in Figure 3b, the clamping position of the bonded square rod is on the K9 glass, which avoids the mechanical stress caused by the clamping of Nd:glass. Without the bonding method, the heat accumulated on the transparent end faces of the neodymium glass can only be dissipated to the side surfaces (water-cooled convective heat transfer coefficient is $500 \mathrm{~W} / \mathrm{m}^{2} \cdot \mathrm{k}$ ), and the heat dissipation of the air in the axial direction of the end face is too low to be noticed (the natural convection heat transfer coefficient of air at room temperature is $\left.5 \mathrm{~W} / \mathrm{m}^{2} \cdot \mathrm{k}\right)$. The thermal conductivity of $\mathrm{K} 9$ glass $(1.4 \mathrm{~W} / \mathrm{m} \cdot \mathrm{k})$ is higher than that of Nd:glass $(0.56 \mathrm{~W} / \mathrm{m} \cdot \mathrm{k})$, which enables Nd:glass to transfer the heat accumulated on the end surface to K9 glass for diffusion, and the overall thermal density of the medium is reduced in the end. As a result, it reduces the thermal stress in the Nd:glass and the risk of its collapse. The two outermost end faces are not deformed due to thermal expansion, so the beam quality can also be improved.

The gain distribution measured in the experiment is shown in Figure 4, the total pump energy is $9.02 \mathrm{~J}$, and the pump pulse width is 500 us. The gain uniformity measured on the entire platform is less than $6.94 \% \mathrm{rms}$ in the $95 \%$ area, and the single-pass small signal gain is about 2.30. The energy storage of the amplifier head is calculated according to Equations (1) and (2) [23].

$$
\begin{gathered}
E_{S}=h v / \gamma \sigma_{l} \\
E_{s t}=\ln \left(G_{0}\right) E_{S} A
\end{gathered}
$$

where $E_{S}$ is the saturated energy storage density; for a four-level system, $\gamma=1 ; \sigma_{l}$ is the emission cross-section of Nd:glass; the calculated saturated energy storage density is $4.97 \mathrm{~J} / \mathrm{cm}^{2} ; G_{0}$ is the single-pass small signal gain obtained from the experiment; $A$ is the gain area; and the calculated energy storage of Nd:glass is $2.65 \mathrm{~J}$.

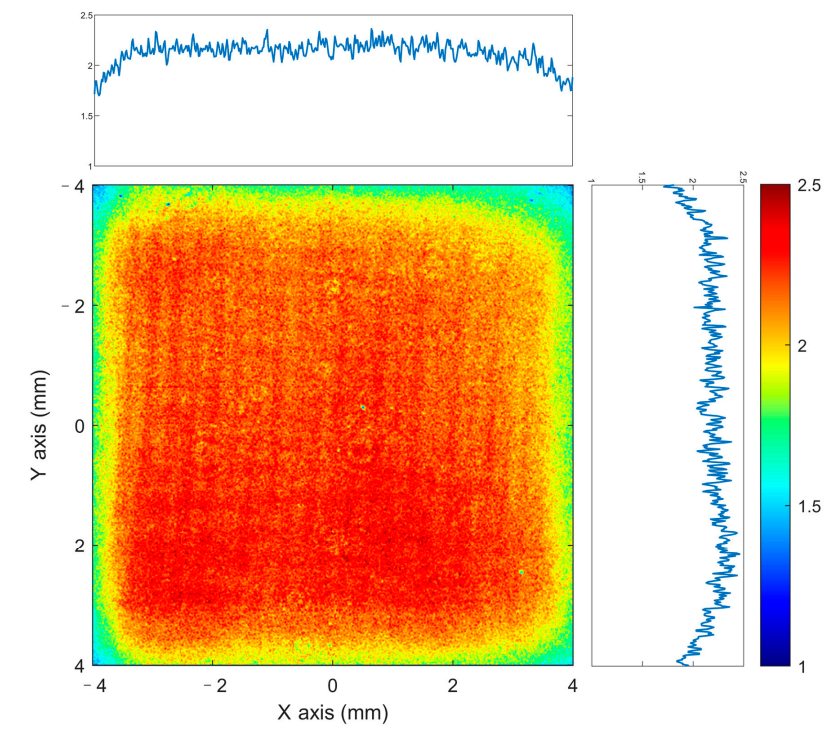

Figure 4. Gain distribution of Nd:glass under total pump energy of $9.02 \mathrm{~J}$.

\section{Experimental Results and Discussion}

\subsection{Thermal Effects}

Under the condition of repetition frequency of $1 \mathrm{~Hz}$ and a pump energy of $9.02 \mathrm{~J}$, the two bonding surfaces of the bonded Nd:glass laser amplifier head gathers a lot of heat, resulting in wavefront aberration. As shown in Figure 5, the thermally induced wavefront difference measured by a wavefront sensor (SID4, Phasics) in the experiment was $0.78 \mu \mathrm{m}$. With the same pumping conditions, the thermally induced wavefront profile difference of the unbonded square-rod Nd:glass of the same specification was measured to be $0.91 \mu \mathrm{m}$. The former one is $85.71 \%$ of the latter one; for this $\mathrm{Nd}$ :glass square rod, the bonding method is not the best for optimizing its thermal effect. A new cooling structure should be tried in later works. 


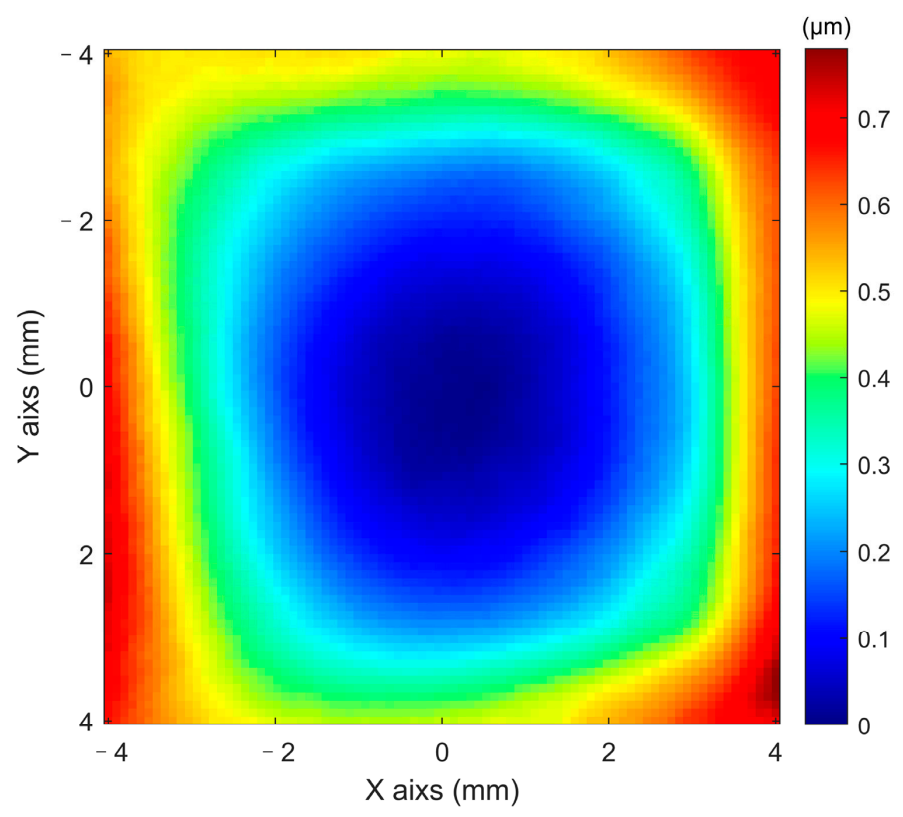

Figure 5. Thermally induced wavefront aberration.

In order to understand the main wavefront types, SID4 was used to decompose the Legendre polynomial of the wavefront aberration in Figure 5. Figure 6 shows the first 21 terms in the analysis of the wavefront aberration's Legendre polynomial coefficient. The fourth, the sixth, and the thirteenth terms are dominant; the fourth and the sixth terms are the defocus of $x$ and $y$, respectively. The thirteenth item was dominant because the gain medium and the pump cross-section were both square, and the distance between the edge of the pump area and the central point was different, resulting in different heat dissipation capabilities. These items can be compensated for by wavefront correctors.

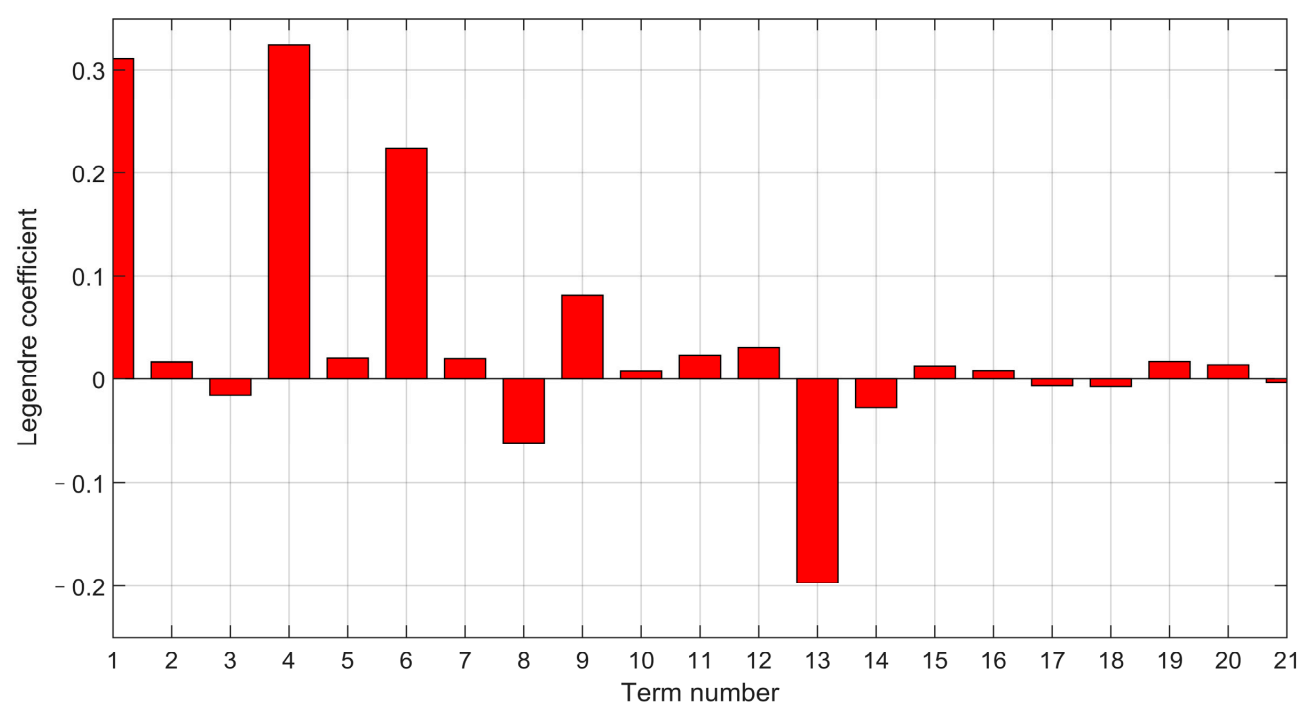

Figure 6. Analysis of the wavefront aberration's Legendre polynomials.

Another function of FR2 in Figure 1 is to compensate for thermally induced depolarization [24]. Figure 7a,b show the near fields of a two-pass laser when the FR2 is not placed and when the FR2 is placed and the depolarization effect is compensated. 
(a)

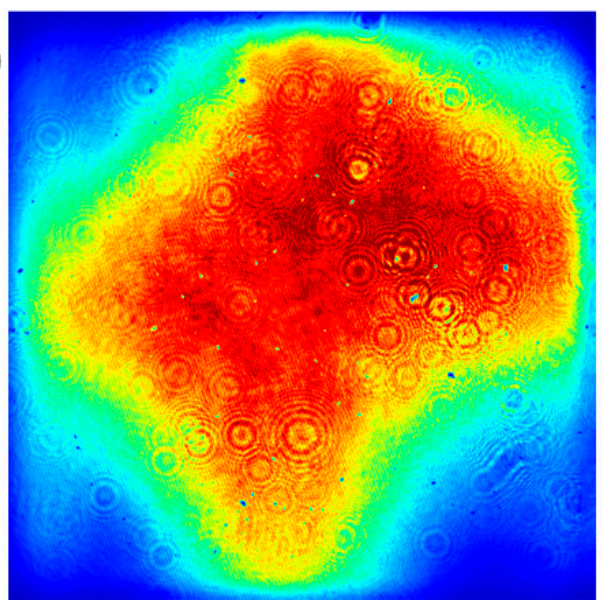

(b)

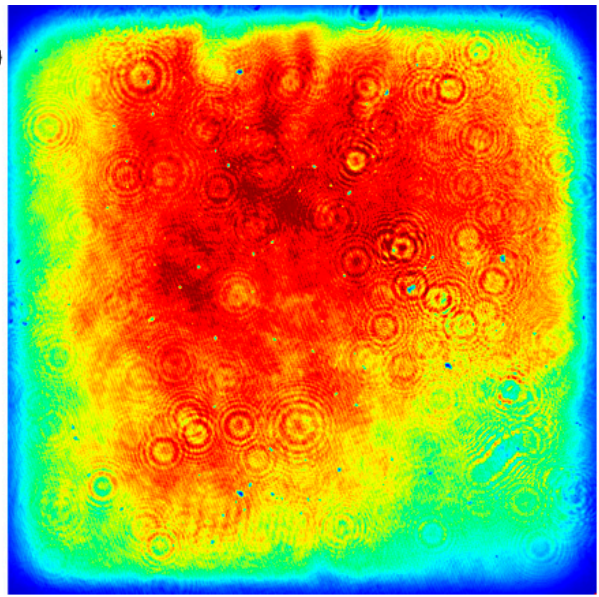

Figure 7. The near fields of a two-pass laser. (a) No depolarization compensation; (b) with depolarization compensation.

\subsection{Output Energy}

Nd:glass has a high energy storage density, but its limited single-pass gain makes it unable to efficiently extract the stored energy during single-pass amplification. Multipass amplification can improve energy extraction efficiency. However, the gain of each pass in the multi-pass amplification gradually decreases due to the extraction of stored energy in the previous pass. The theoretical calculation of the multi-pass amplification output energy is based on the iterative calculation [25], and Equations (3)-(5) are the main calculation formulas:

$$
\begin{gathered}
E_{\text {out }}=T \cdot E_{s} \cdot \ln \left\{1+\left[\exp \left(\frac{E_{\text {in }}}{E s}\right)-1\right] \cdot \exp \left(g_{0} l\right)\right\}, \\
\eta_{l}=\left(E_{\text {out }}-E_{\text {in }}\right) / g_{0} l E s, \\
g_{0}^{\prime}=\left(1-\eta_{l}\right) g_{0},
\end{gathered}
$$

where $E_{\text {in }}$ is the energy injected in a certain pass; $E_{\text {out }}$ is the energy of the single-pass amplification; $E_{s}$ is the saturated energy storage density; $g_{0}$ is the single-pass small signal gain coefficient; and $l$ is the length of the gain zone; the average single-pass transmittance $T$ of the amplifier obtained by experimental measurement is $85.04 \% ; \eta_{l}$ is the single-pass extraction efficiency; and $g_{0}^{\prime}$ is the next single-pass small signal gain coefficient. After many iterations of calculation, the output energy curves of differently injected laser energy in multi-pass amplification are shown in Figure 8. The experimental measurement results and the theoretical calculation results were in good agreement in the four-pass, eight-pass, and twelve-pass amplification. A sampling mirror was used to sample the amplified laser energy and reflect it to the energy harvester (Gentec QE65S) because the output energy was relatively large. For the four-pass and eight-pass amplification, when the injected laser energy was $6.50 \mathrm{~mJ}$, energies of $83.86 \mathrm{~mJ}$ and $704.72 \mathrm{~mJ}$ were obtained, respectively, and neither reached gain saturation. For the twelve-pass amplification, when the injected energy was $3.00 \mathrm{~mJ}$, the gain saturation was reached, and the maximum output energy was $1.17 \mathrm{~J}$. The energy extraction efficiency reached $44.23 \%$. The optical-to-optical efficiency reached $13.01 \%$. 


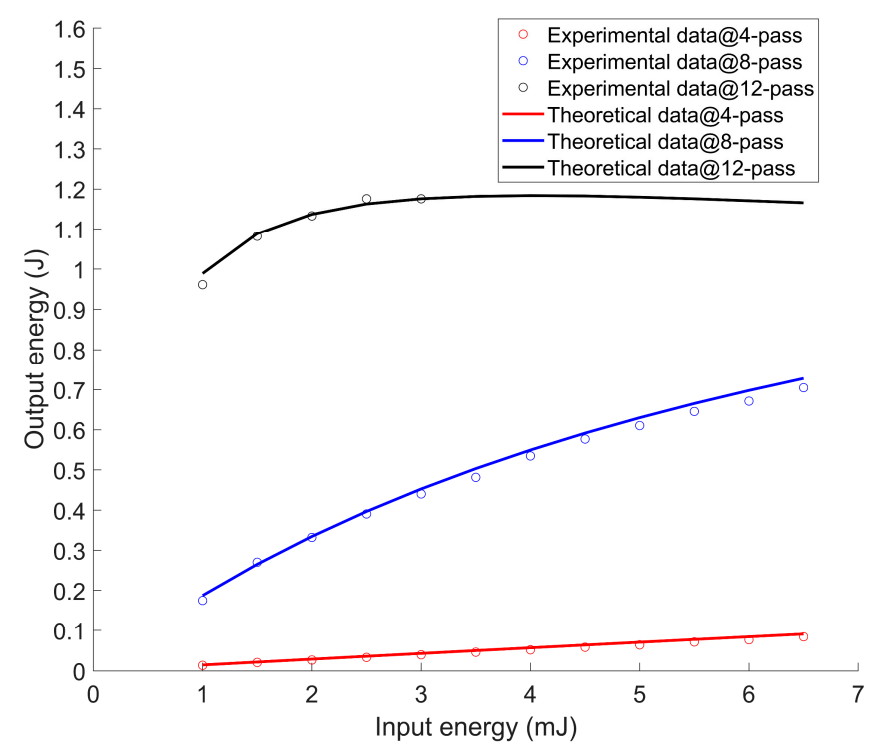

Figure 8. Multi-pass amplification energy comparison between theoretical calculations and experimental results.

\subsection{Beam Quality}

Figure 9 shows the twelve-pass near-field profile at the laser image plane position with a repetition frequency of $1 \mathrm{~Hz}$ and an output energy of $1.17 \mathrm{~J}$ by using a CCD (Charge Coupled Device) camera (Camyu Corp., GYD-SG1024B12GA). Some clear diffraction rings in the image were caused by some dead pixels in the measurement system. The modulation degree of the laser intensity within the range of $90 \%$ was 1.42 . Because the Pockels cell produced a few small damage points in the previous experiment, it accumulated during the twelve-pass amplification process. The rings appearing in Figure 9 are diffraction phenomena caused by some dust in the diagnostic channel. In subsequent research, a spatial light modulator should be used for beam shaping to improve modulation.

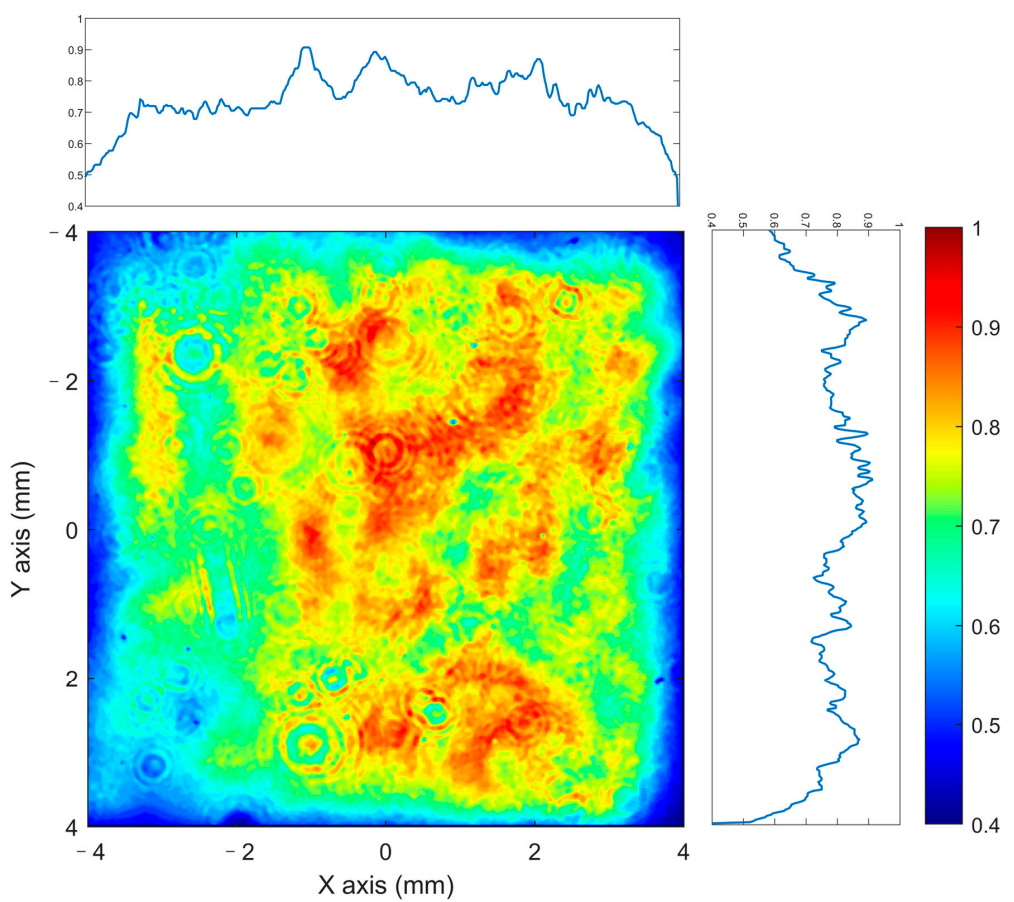

Figure 9. The near-field profile with a twelve-pass amplification output energy of $1.17 \mathrm{~J}$. 
The corresponding far-field mode and the far-field energy concentration measured at the focal length of the lens are shown in Figure 10. The far-field energy concentration was $81.88 \%$, at 2.5 times the diffraction limit. There are some speckles around the spot, which reduces the energy concentration. Some of the defocus aberrations can be compensated for by adjusting the lens position in the laser-magnifying cavity, and the remaining aberrations require further improvement and optimization by a wavefront corrector.

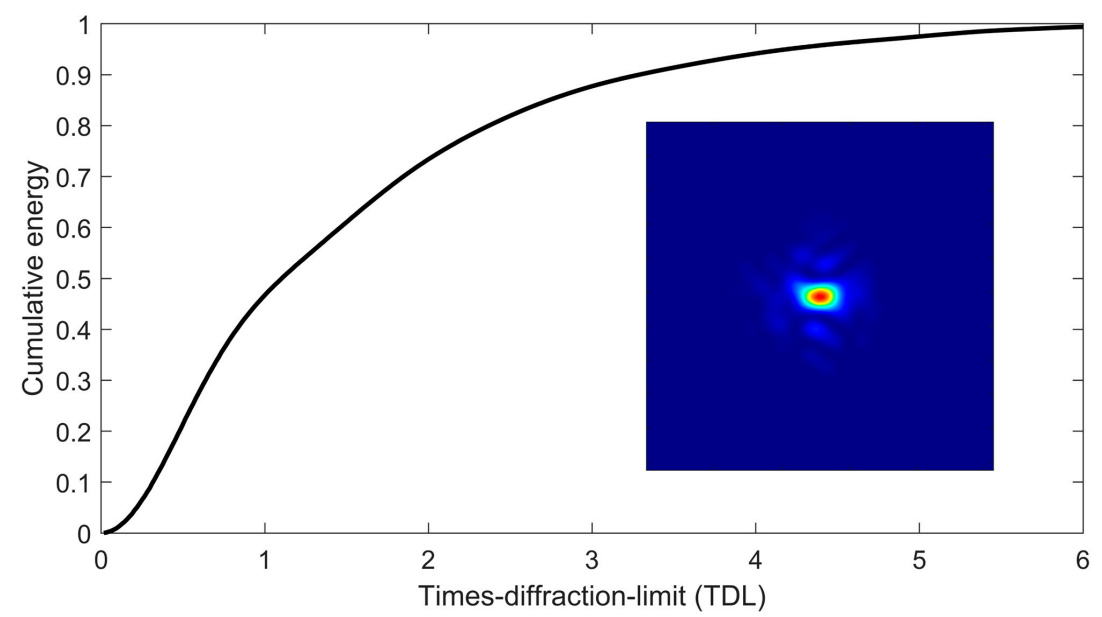

Figure 10. The far-field mode and energy concentration with a twelve-pass amplification output energy of $1.17 \mathrm{~J}$.

\section{Conclusions}

In this paper, a twelve-pass amplification system with diode end-pumped bonded $\mathrm{Nd}$ :glass to achieve Joule-level output energy is described. Under the $9.02 \mathrm{~J}$ pump energy at $802 \mathrm{~nm}, 1.17 \mathrm{~J}$ saturated output energy was achieved at a repetition frequency of $1 \mathrm{~Hz}$ and $3.00 \mathrm{~mJ}$ energy injection. The optical-to-optical efficiency of $13.01 \%$ and the effective energy extraction efficiency of $44.23 \%$ are higher than those of the existing similar laser systems. In addition, the single-pass thermal wavefront aberration was $0.78 \mu \mathrm{m}$, which is $85.71 \%$ of the non-bonded square-rod $\mathrm{Nd}$ :glass under the same conditions. In the case of the maximum output energy of the twelve-pass amplification system, the near-field modulation of the beam was 1.42 within the $90 \%$ spot range, and the far-field energy concentration was $81.88 \%$ at the 2.5 -fold diffraction limit. In the subsequent works, a spatial light modulator and a wavefront corrector will be used to improve the quality of the laser beam. Experiments show that the Joule-level bonded Nd:glass laser amplification system with high optical efficiency has potential application prospects in high-power laser amplification systems and can be used as a pump source in various systems, e.g., in optical parametric chirped pulse amplification (OPCPA) systems.

Author Contributions: Conceptualization, W.H. and J.W.; Formal analysis, L.P. and S.J.; Funding acquisition, X.L. (Xuechun Li) and J.Z.; Investigation, J.Z.; Methodology, L.P., W.H., J.W. and W.F.; Project administration, J.W. and X.L. (Xuechun Li); Resources, X.L. (Xinghua Lu) and W.F.; Software, J.G.; Writing—original draft, L.P.; Writing—review \& editing, S.J., W.H., J.G., X.L. (Xinghua Lu), J.W., W.F., X.L. (Xuechun Li) and J.Z. All authors have read and agreed to the published version of the manuscript.

Funding: This research was funded by the Strategic Priority Research Program of the Chinese Academy of Sciences (grant No. XDA25020307) and the Program of Shanghai Academic Research Leader (19XD1404000).

Institutional Review Board Statement: No applicable.

Informed Consent Statement: No applicable.

Conflicts of Interest: The authors declare no conflict of interest. 


\section{References}

1. Zhu, J.; Zhu, J.; Li, X.; Zhu, B.; Ma, W.; Lu, X.; Fan, W.; Liu, Z.; Zhou, S.; Xu, G.; et al. Status and development of high-power laser facilities at the NLHPLP. High Power Laser Sci. Eng. 2018, 6. [CrossRef]

2. Hogan, W.; Moses, E.; Warner, B.; Sorem, M.; Soures, J. The National Ignition Facility. Nucl. Fusion 2001, 41, 567-573. [CrossRef]

3. Zhao, N.; Jiao, J.; Xie, D.; Zhou, H.; Zhang, S.; Lang, Y.; Zou, D.; Zhuo, H. Near- $100 \mathrm{MeV}$ proton acceleration from $1021 \mathrm{~W} / \mathrm{cm}^{2}$ laser interacting with near-critical density plasma. High Energy Density Phys. 2020, 37, 100889. [CrossRef]

4. Fu, W.; Liang, E.P.; Fatenejad, M.; Lamb, D.Q.; Grosskopf, M.; Park, H.-S.; Remington, B.; Spitkovsky, A. Increase of the density, temperature and velocity of plasma jets driven by a ring of high energy laser beams. High Energy Density Phys. 2013, 9, 336-340. [CrossRef]

5. Ciardi, A.; Vinci, T.; Fuchs, J.; Albertazzi, B.; Riconda, C.; Pépin, H.; Portugall, O. Astrophysics of Magnetically Collimated Jets Generated from Laser-Produced Plasmas. Phys. Rev. Lett. 2013, 110. [CrossRef] [PubMed]

6. Danson, C.N.; Haefner, C.; Bromage, J.; Butcher, T.; Chanteloup, J.-C.F.; Chowdhury, E.A.; Galvanauskas, A.; Gizzi, L.A.; Hein, J.; Hillier, D.I.; et al. Petawatt and exawatt class lasers worldwide. High Power Laser Sci. Eng. 2019, 7. [CrossRef]

7. Su, H.; Peng, Y.; Chen, J.; Li, Y.; Wang, P.; Leng, Y. A High-Energy, 100 Hz, Picosecond Laser for OPCPA Pumping. Appl. Sci. 2017, 7, 997. [CrossRef]

8. Bromage, J.; Bahk, S.-W.; Begishev, I.A.; Dorrer, C.; Guardalben, M.J.; Hoffman, B.N.; Oliver, J.B.; Roides, R.G.; Schiesser, E.M.; Iii, M.J.S.; et al. Technology development for ultraintense all-OPCPA systems. High Power Laser Sci. Eng. 2019, 7. [CrossRef]

9. Kalentics, N.; Boillat, E.; Peyre, P.; Ćirić-Kostić, S.; Bogojević, N.; Logé, R.E. Tailoring residual stress profile of Selective Laser Melted parts by Laser Shock Peening. Addit. Manuf. 2017, 16, 90-97. [CrossRef]

10. Gallais, L.; Commandré, M. Laser-induced damage thresholds of bulk and coating optical materials at $1030 \mathrm{~nm}, 500 \mathrm{fs}$. Appl. Opt. 2013, 53, A186-A196. [CrossRef] [PubMed]

11. Banerjee, S.; Mason, P.D.; Ertel, K.; Phillips, P.J.; De Vido, M.; Chekhlov, O.; Divoky, M.; Pilar, J.; Smith, J.; Butcher, T.; et al. 100 J-level nanosecond pulsed diode pumped solid state laser. Opt. Lett. 2016, 41, 2089-2092. [CrossRef] [PubMed]

12. Mason, P.; Divoký, M.; Ertel, K.; Pilař, J.; Butcher, T.; Hanuš, M.; Banerjee, S.; Phillips, J.; Smith, J.; De Vido, M.; et al. Kilowatt average power $100 \mathrm{~J}$-level diode pumped solid state laser. Optica 2017, 4, 438-439. [CrossRef]

13. Ogino, J.; Tokita, S.; Kitajima, S.; Yoshida, H.; Li, Z.; Motokoshi, S.; Morio, N.; Tsubakimoto, K.; Fujioka, K.; Kodama, R.; et al. 10 J operation of a conductive-cooled Yb:YAG active-mirror amplifier and prospects for $100 \mathrm{~Hz}$ operation. Opt. Lett. 2021, 46, 621-624. [CrossRef] [PubMed]

14. Hornung, M.; Liebetrau, H.; Keppler, S.; Kessler, A.; Hellwing, M.; Schorcht, F.; Becker, G.A.; Reuter, M.; Polz, J.; Körner, J.; et al. $54 \mathrm{~J}$ pulses with $18 \mathrm{~nm}$ bandwidth from a di-ode-pumped chirped-pulse amplification laser system. Opt. Lett. 2016, 41, 5413-5416. [CrossRef] [PubMed]

15. Haefner, C.L.; Bayramian, A.; Betts, S.; Bopp, R.; Buck, S.; Cupal, J.; Drouin, M.; Erlandson, A.; Horáček, J.; Horner, J.; et al. High average power, diode pumped petawatt laser systems: A new generation of lasers enabling precision science and commercial applications. In Proceedings of the SPIE Research Using Extreme Light: Entering New Frontiers with Petawatt-Class Lasers III, Prague, Czech Republic, 26 June 2017.

16. Huang, T.; Huang, W.; Wang, J.; Lu, X.; Pan, X.; Guo, J.; Fan, W.; Li, X. High energy diode-pumped sapphire face-cooled Nd:glass multi-slab amplifier. Opt. Laser Technol. 2018, 107, 415-423. [CrossRef]

17. Yao, K.; Xie, X.; Tang, J.; Fan, C.; Gao, S.; Lu, Z.; Chen, Z.; Xue, Q.; Zheng, K.; Zhu, Q. Diode-side-pumped joule-level square-rod $\mathrm{Nd}$ :glass amplifier with $1 \mathrm{~Hz}$ repetition rate and ultrahigh gain. Opt. Express 2019, 27, 32912-32923. [CrossRef] [PubMed]

18. Takagi, H.; Kikuchi, K.; Maeda, R.; Chung, T.R.; Suga, T. Surface activated bonding of silicon wafers at room temperature. Appl. Phys. Lett. 1996, 68, 2222-2224. [CrossRef]

19. Traggis, N.; Claussen, N. Epoxy free bonding for high performance lasers. In Proceedings of the 11th Annual Directed Energy Symposium Pro-ceedings; Directed Energy Professional Society: Albuquerque, NM, USA, 2008.

20. Wang, G.; Sun, X.; Xu, J.; Shan, Y.; Han, X.; Xu, J.; Li, J. Pressureless thermal diffusion bonding of transparent AlON ceramics by using a powder interlayer of parent material. Scr. Mater. 2019, 171, 118-121. [CrossRef]

21. Nagisetty, S.S.; Severova, P.; Miura, T.; Smrž, M.; Kon, H.; Uomoto, M.; Shimatsu, T.; Kawasaki, M.; Higashiguchi, T.; Endo, A. Lasing and thermal characteristics of Yb:YAG/YAG composite with atomic dif-fusion bonding. Laser Phys. Lett. 2017, 14, 015001. [CrossRef]

22. Mukhin, I.; Perevezentsev, E.; Palashov, O. Fabrication of composite laser elements by a new thermal diffusion bonding method. Opt. Mater. Express 2014, 4, 266-271. [CrossRef]

23. Koechner, W. Solid-State Laser Engineering, 6th ed.; Springer: Berlin/Heidelberg, Germany, 2006.

24. Guo, J.; Wang, J.; Wei, H.; Huang, W.; Huang, T.; Xia, G.; Fan, W.; Lin, Z. High-power, Joule-class, temporally shaped multi-pass ring laser amplifier with two Nd:glass laser heads. High Power Laser Sci. Eng. 2019, 7. [CrossRef]

25. Frantz, L.M.; Nodvik, J.S. Theory of Pulse Propagation in a Laser Amplifier. J. Appl. Phys. 1963, 34, 2346-2349. [CrossRef] 